\title{
Advanced trends in magnetic resonance imaging in assessment of lumbar intervertebral degenerative disk disease
}

\author{
Rania Sobhy Abou Khadrah ${ }^{1 *}$ D, Mohamed F. Dawoud ${ }^{1}$, Ashraf Ali Abo-Elsafa ${ }^{2}$ and Amr M. Elkilany ${ }^{1}$
}

\begin{abstract}
Background: T2 mapping and DWI are newly quantitated method for disk degeneration assessment; they were used in the determination of an early stage of intervertebral disk degeneration. T2 mapping was quantitatively sensitive for detecting the early stage and aging-related changes in intervertebral disk degeneration. Furthermore, T2 mapping and apparent diffusion coefficient values (ADC) in lumbar intervertebral disks indirectly correlated with the Pfirrmann grades in IVDD and age-related disk degeneration. The aim of this study is to evaluate the sensitivity of T2 mapping and apparent diffusion coefficient in the determination of an early stage of intervertebral disk degeneration.
\end{abstract}

Results: $T 2$ relaxometry values were found to decrease with the increased disk degeneration except in grade $\mathrm{V}$ where it was found to be increased again. There was a negative correlation between T2 values and semiquantitative grading (Pfirrmann Grading) of disk degeneration and T2 values were significantly different when comparing grade I to V. A T2 value of nucleus pulposus (NP) was more sensitive than annulus fibrosus (AF) and entire of the disk. ADC values were found to decrease with the increased degree of disk degeneration; there was a weakly significant negative correlation between age and T2 mapping values, ADC values of nucleus pulposus, and entire of disk.

Conclusion: T2 mapping was significantly different when comparing grade I to $\mathrm{V}$ while ADC value had a significant weak negative correlation with age, so T2 mapping and to a little extent ADC can be used for quantitative analysis of early disk generation seeking for early diagnosis and better management.

Keyword: Intervertebral degenerative disk (IVDD), T2 mapping, Diffusion-weighted image (DWI), Apparent diffusion coefficient (ADC), Pfirrmann grading

\section{Background}

Lower-back pain was considered as one of the most common causes of limitation of activity in patients younger than 45 years. The lifelong prevalence of lower-back pain is up to $80 \%$, leading to countless costs due to treatment and work absence [1]. Degenerative disk disease (DDD) was regarded as the most common cause of lower-back pain [2].

Intervertebral disks formed of two distinct components. The nucleus pulposus was well hydrated; incompressible with gelatinous consistency surrounded by a more rigid ring of fibrous collagen called the annulus

\footnotetext{
* Correspondence: rotinia2009@gmail.com

${ }^{1}$ Radiology and Medical Imaging Department, Faculty of Medicine, Tanta

University, Tanta, Egypt

Full list of author information is available at the end of the article
}

fibrosus disks in young health individuals were usually well hydrated. Degeneration of the disk was best seen on T2W images [3, 4].

Piffirmann et al. [5] were classified disk degeneration based on combination of a lot of factors as: disk structure, ability to differentiate between the nucleus pulposus and the annulus fibrosus, signal pattern, and the height of intervertebral disk [6].

Standard conventional magnetic resonance (MR) protocols of the spine include sagittal T1-weighted spin-echo (SE) or fast spin-echo (FSE); sagittal and axial T2weighted FSE sequences were used for evaluation of the disks structures but we were in need for a quantitative method to evaluate the molecular composition and structural integrity $[7,8]$. 
Recently, multiple quantitative MRI techniques had been used for evaluating the intervertebral disk, combined application of T2 mapping and diffusion-weighted image (DWI) as both of them related to proteoglycan and water content of the disk had potential value in improving diagnostic sensitivity and accuracy [9].

T2 mapping had been used to assess the spine as it had the ability to evaluate quantitatively the deterioration of the molecular composition and integrity of structure of the intervertebral disks [10]. T2 relaxation times sequence was firstly used to evaluate the knee articular surface and then trials made to apply it to the intervertebral disk (IVD) as it was highly sensitive to water content and the arrangement of the collagen structure. It also affected by the dipolar interaction between the disk molecules because of the anisotropic motion of water molecules in the collagen matrix [11].

Another promising tool for noninvasive disks molecular evaluation was diffusion-weighted image (DWI) as the spinal disk was an avascular structure and depended on diffusion for its nutrition, so the degenerative disk disease may affect molecular diffusibility $[12,13]$.

T2 and apparent diffusion coefficient (ADC) mappings are two newly quantitative MR imaging tools used for assessing early disk degeneration [14]. We tried to study the relationship between both imaging tools and intervertebral disk degeneration (IVDD) aiming for early diagnosis and hence improve management as early detection of disk degeneration will improve the treatment and may prevent invasive management techniques.

\section{Methods}

\section{Patient populations}

Prospective study was conducted upon 60 patients and 30 normal volunteers (control group) with nearly the same sex and age that were referred to the MRI unit. Patients were examined in the period between May 2017 and May 2018. The study was approved by the hospital ethical committee and all patients and volunteers agreed to participate in the study.

\section{Inclusion criteria}

Patients aged more than 20 years with the presence of significant low back pain, leg numbness, and tingling for more than 3 months severe enough to require medical consultation or treatment. Volunteers were included aged more than 20 years and had no symptom with normal morphological criteria of the disks on conventional MRI study.

\section{Exclusion criteria}

Patients younger than 20 years, patients with IVD infection whatever the etiology, patients with blood disease affecting the vertebra, spine tumor and previous back surgery, and any abnormal signals affecting the vertebra or the IVD were excluded. Any contraindication to MRI (patients had any metallic prosthesis as a pacemaker and cochlear implants).

\section{Patients preparations}

Patients were instructed to remove all ferromagnetic objects like metallic objects and were instructed about the importance of being calm with no motion throughout the time of examination. We obtained informed consent from all patients and healthy volunteers. Any expected risk was explained, and the privacy of all patients' data was guaranteed. Proper history taking was done as regard name, age, sex, job, and present symptoms as low back pain and duration of pain.

\section{Image acquisition}

All patients were subjected to an MRI examination performed by 1.5 Tesla G.E Machine. Patients were instructed to remain in the supine position with their legs extended and relaxed. Spin-echo sequences were acquired in the sagittal plane to generate quantitative T2 mapping; to remove the possible role of diurnal disk hydration changes, all studied cases underwent imaging in the afternoon. MR scanning sequences were T2WI, sagittal T2 mapping and DWI, ADC map, and quantitative DWI analysis (ADC measurement).T2WI was done in the sagittal plane with the following parameter: TR 4.00 ms, TE $102 \mathrm{~ms}$, slice thickness $5 \mathrm{~mm}$, field of view 28 $\mathrm{cm}$, matrix $512 \times 512$. DWI single-shot, spin-echo echoplanar imaging (SE-EPI) sequences with fat suppression were utilised with TR/TE $=4.00 / 63 \mathrm{~ms}$. Diffusion gradients of two $b$ values, $b 0-0$ and $b=600 \mathrm{~s} / \mathrm{mm}$. Field of view $28 \mathrm{~cm}$, slice thickness $5 \mathrm{~mm}$, matrix 256× 256. T2 mapping was obtained in sagittal view with the following parameter $\mathrm{TR} / \mathrm{TE}=1000 / 8 \mathrm{~ms}$, the number of excitations $(\mathrm{NEX})=2$, flip angle $180^{\circ}$, matrix $256 \times 256$, slice thickness $5 \mathrm{~mm}$.

\section{Data analysis}

Five lumbar disks (L1/L2-L5/S1) in each case were firstly classified with visual inspection using the midsagittal T2W image as a reference. The criteria set forth by Pfirnmann et $\mathrm{al}^{\cdot}$ [5] (Grade I: normal to grade V: advanced degeneration with collapsed disk space) were as follows:

- Grade I: disk is homogeneous with bright hyperintense white signal intensity and normal disk height

- Grade II

- Disk is inhomogeneous, but keeping the hyperintense white signal 
- Nucleus and annulus are clearly differentiated, and a gray horizontal band could be present

- Disk height is normal

- Grade III

- Disk is inhomogeneous with an intermittent gray signal intensity

- Distinction between nucleus and annulus is unclear

- Disk height is normal or slightly decreased

- Grade IV

- Disk is inhomogeneous with a hypointense dark gray signal intensity

- There is no more distinction between the nucleus and annulus

- Disk height is slightly or moderately decreased

- Grade V

- Disk is inhomogeneous with a hypointense black signal intensity

- There is no more difference between the nucleus and annulus

- The disk space is collapsed
All images were graded by two experienced radiologists (10 and 15 years of experience with MRI). Both observers had experienced in musculoskeletal MRI regions of interest (ROI) selection (at least 1 year experience). Both observers 1 and 2 repeated the same analysis to evaluate intraobserver agreement 1 month apart independently. Observers graded disks according to the improved Pfirrmann IVD degeneration grading standard.

\section{Data processing and post-processing}

Diffusion-weighted imaging and T2 mapping were transferred to the workstation (advantage window 4, 7) where they converted to color-coded map images automatically by the loaded program depending on the color mapping images. In T2 mapping, healthy nucleus pulpous usually appear blue and annulus fibrosus appear green and mean values for the regions of interest were set as T2 values.

Five lumbar intervertebral disks were manually segmented, encompassing the complete nucleus pulposus

Table 1 Relation between PG and different parameters $(n=300)$

\begin{tabular}{|c|c|c|c|c|c|c|c|c|}
\hline & & \multicolumn{5}{|l|}{$P G$} & \multirow[t]{2}{*}{$F$} & \multirow[t]{2}{*}{$p$} \\
\hline & & $1(n=34)$ & $2(n=72)$ & $3(n=86)$ & $4(n=86)$ & $5(n=22)$ & & \\
\hline \multirow[t]{12}{*}{ T2 mapping } & Central & & & & & & & \\
\hline & Min.-Max. & 91.0-180.0 & $77.0-184.0$ & $64.0-154.0$ & $54.0-110.0$ & $66.0-166.0$ & $27.003^{*}$ & $<0.001^{*}$ \\
\hline & Mean \pm SD. & $133.06 \pm 32.85$ & $124.50 \pm 24.02$ & $102.49 \pm 23.24$ & $77.53 \pm 14.55$ & $109.18 \pm 33.82$ & & \\
\hline & Median & 122.0 & 125.50 & 102.0 & 78.0 & 98.0 & & \\
\hline & Peripheral & & & & & & & \\
\hline & Min.-Max. & $56.0-86.0$ & $56.0-144.0$ & $52.0-147.0$ & $45.0-95.0$ & $56.0-184.0$ & $6.905^{*}$ & $<0.001^{*}$ \\
\hline & Mean \pm SD. & $74.71 \pm 7.97$ & $82.06 \pm 19.98$ & $76.02 \pm 20.46$ & $65.28 \pm 13.32$ & $96.55 \pm 41.63$ & & \\
\hline & Median & 72.0 & 78.50 & 71.0 & 65.0 & 78.0 & & \\
\hline & All & & & & & & & \\
\hline & Min.-Max. & 77.0-121.0 & $77.0-137.0$ & $65.0-138.0$ & $56.0-96.0$ & $70.0-144.0$ & $19.291^{*}$ & $<0.001^{*}$ \\
\hline & Mean \pm SD & $101.12 \pm 13.27$ & $102.69 \pm 14.32$ & $89.81 \pm 16.80$ & $75.49 \pm 11.70$ & $101.82 \pm 26.29$ & & \\
\hline & Median & 98.0 & 102.0 & 88.0 & 75.0 & 100.0 & & \\
\hline \multirow[t]{12}{*}{ Diffusion } & $C$ & & & & & & & \\
\hline & Min.-Max. & $1.57-2.04$ & $1.43-2.70$ & $1.01-2.21$ & $1.15-2.60$ & $1.10-1.87$ & $8.018^{*}$ & $<0.001^{*}$ \\
\hline & Mean \pm SD & $1.78 \pm 0.16$ & $1.82 \pm 0.29$ & $1.72 \pm 0.25$ & $1.54 \pm 0.24$ & $1.47 \pm 0.28$ & & \\
\hline & Median & 1.80 & 1.74 & 1.81 & 1.51 & 1.52 & & \\
\hline & P & & & & & & & \\
\hline & Min.-Max. & $1.08-2.22$ & $1.02-2.12$ & $1.09-2.54$ & $1.01-2.70$ & $1.08-1.58$ & $3.303^{*}$ & $0.013^{*}$ \\
\hline & Mean \pm SD. & $1.54 \pm 0.32$ & $1.55 \pm 0.25$ & $1.62 \pm 0.31$ & $1.51 \pm 0.30$ & $1.27 \pm 0.16$ & & \\
\hline & Median & 1.55 & 1.59 & 1.57 & 1.46 & 1.30 & & \\
\hline & All & & & & & & & \\
\hline & Min.-Max. & $1.40-1.84$ & $1.24-2.40$ & $1.0-2.10$ & $1.12-2.45$ & $1.11-1.58$ & $8.036^{*}$ & $<0.001^{*}$ \\
\hline & Mean \pm SD. & $1.62 \pm 0.14$ & $1.65 \pm 0.25$ & $1.61 \pm 0.22$ & $1.47 \pm 0.27$ & $1.28 \pm 0.15$ & & \\
\hline & Median & 1.59 & 1.63 & 1.61 & 1.42 & 1.28 & & \\
\hline
\end{tabular}

F: for ANOVA test, $\mathrm{p}: p$ value for association between PG and different parameters 


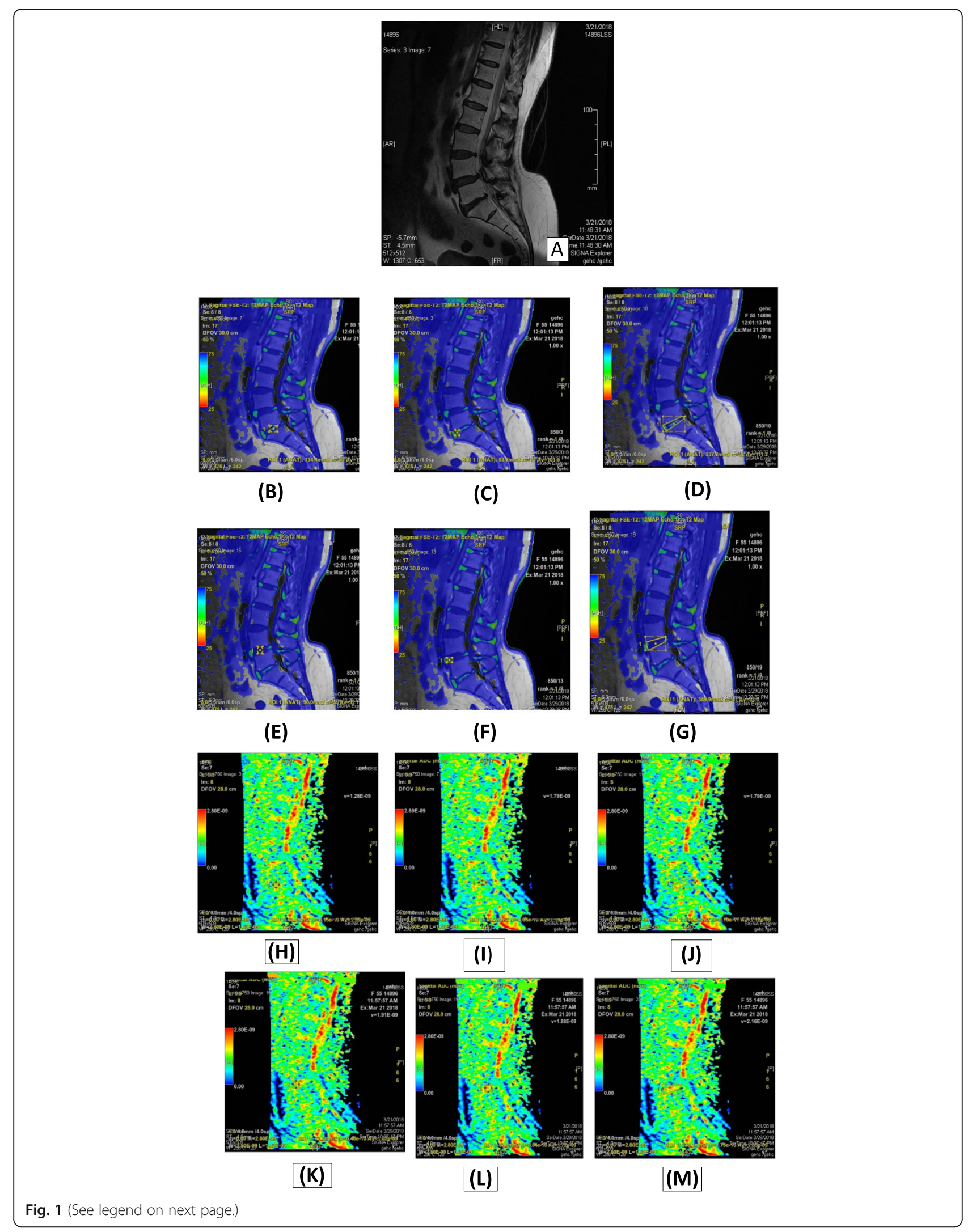


(See figure on previous page.)

Fig. 1 Female patient 55 years old presented with low back pain referred to left leg for 9 months. a Sagittal T2Wl of lumbar spine showing: L3/L4 heterogeneous, intermediate signal intensity, unclear nucleus-annulus distinction, normal to slightly decreased disk height (grade 3). L4/L5 Heterogeneous, intermediate to hypointense signal, lost nucleus-annulus distinction, moderately decreased disk space (grade 4). L5/S1 heterogeneous, hypointense signal, lost nucleus-annulus distinction, collapsed disk space (grade 5). Sagittal color coded post processing T2 mapping (b-d) and $(\mathbf{e}-\mathbf{g})$ of most degenerated disks L4-5 and L5-S1 respectively, sagittal post processing ADC mapping (h-j) and (k-m) of same respectively with ROls placed on nucleus pulposus, annulus fibrosus and entire of the disks and the values tabulated as follows:

\begin{tabular}{|c|c|c|c|c|c|c|}
\hline & \multicolumn{3}{|c|}{ T2 mapping } & \multicolumn{3}{|c|}{ ADC values } \\
\hline & $N P$ & $\mathrm{AF}$ & Entire of disk & $N P$ & $\mathrm{AF}$ & Entire of disk \\
\hline $\mathrm{L} 1 / \mathrm{L} 2$ & 106 & 69 & 94 & 1.6 & 1.57 & 1.59 \\
\hline L2/L3 & 139 & 82 & 110 & 1.95 & 1.58 & 1.70 \\
\hline L3/L4 & 100 & 99 & 103 & 1.59 & 1.44 & 1.41 \\
\hline$L 4 / L 5$ & 91 & 87 & 91 & 1.72 & 1.62 & 1.63 \\
\hline L5/S1 & 98 & 78 & 86 & 1.19 & 1.59 & 1.12 \\
\hline
\end{tabular}

and annulus fibrosus and taking care not to segment the subchondral bone and vertebral endplate.

In midsagittal slice, areas of interest were delineated along the disk contours by application of the spherical regions of interest tool. The nucleus region of interest was placed in the most central area of the disk. The annulus regions of interest were positioned in the most anterior portions of the disk. A clear separation of nucleus pulposus and outer annulus fibrosus was not possible especially for degenerated disks.

All regions of interest were selected on the conventional images and transferred via copy and paste into the T2 maps ADC map. ADC maps were calculated automatically with the MRI system and ADC values were expressed in square millimeters per second.

\section{Statistical analyses}

All statistical analyses were performed using SPSS Version 2. T2 values and ADC values were statistically described in terms of mean \pm standard deviation $( \pm S D)$. One-way ANOVA was used to detect significance between $\mathrm{ADC}$ values and $\mathrm{T} 2$ relaxation values of different degeneration grades. Pearson correlation coefficient was utilized to measure the correlation between ADC values and T2 values with Pfirmann grade. Linear regression analysis used to correlate $\mathrm{T} 2$ relaxation values and ADC values with age. Finally, receiver operating characteristic ( $\mathrm{ROC}$ ) curves were drawn and the area under the curves (AUC) and the cut-off values were calculated; $p<0.05$ was considered statically significant.

\section{Results}

This study was conducted upon 60 patients (30 males and 30 females) and 30 healthy volunteers (20 male and ten females); patient's age ranged between 35 and 62 years with mean age $47.5 \pm 7.8$ years while volunteer's age were 33-55 years. Both patients and volunteers performed conventional MRI lumbar spine and complementary sagittal T2 mapping and diffusion-weighted imaging. No statically significant difference was observed between the age and sex of both patients and volunteers ( $p$ value > $0.05)$, the inter- and intraobserver reliability between the two readers 1 and 2 who draw the (ROI) were non-significant in T2 mapping and DWI; there was significant difference ( $p$ value $<0.001$ ) between the patients and control group in both central and all disk T2 mappings and DWI, while no significant difference in the peripheral part of the disk in both T2 mapping and DWI with ( $p$ value 0.013 and 0.43 ) respectively.

\section{Descriptive analyses of the studied disks}

The whole number of pathological disks were 300; T2-weighted image-based Pfirrmann grade classification of IVD disks were Pfirrmann grades PG I 34 (11.3\%), PG II 72 (24.0\%), PG III 86 (28.7\%), PG IV 86 (28.7\%), and PG V 22 (7.3\%).

\section{T2 mapping and ADC mapping findings}

In T2 mapping, the nucleus pulposus T2 relaxometry values were decreased with increasing Pfirmann grades and $\mathrm{T} 2$ values were significantly different when comparing grade I to $\mathrm{V}$ with $p<0.001$ using one-way ANOVA (Table 2). Also statistically significant difference was found between the $\mathrm{T} 2$ relaxation time of annulus fibrosus and entire disk between different grades with $p<0.001$ using one-way ANOVA (Table 1 and Fig. 1).

In the nucleus pulposus, annulus fibrosus, entire of the disk ADC values were inversely correlated with Pfirrmann grades and $A D C$ values were significantly variable when 


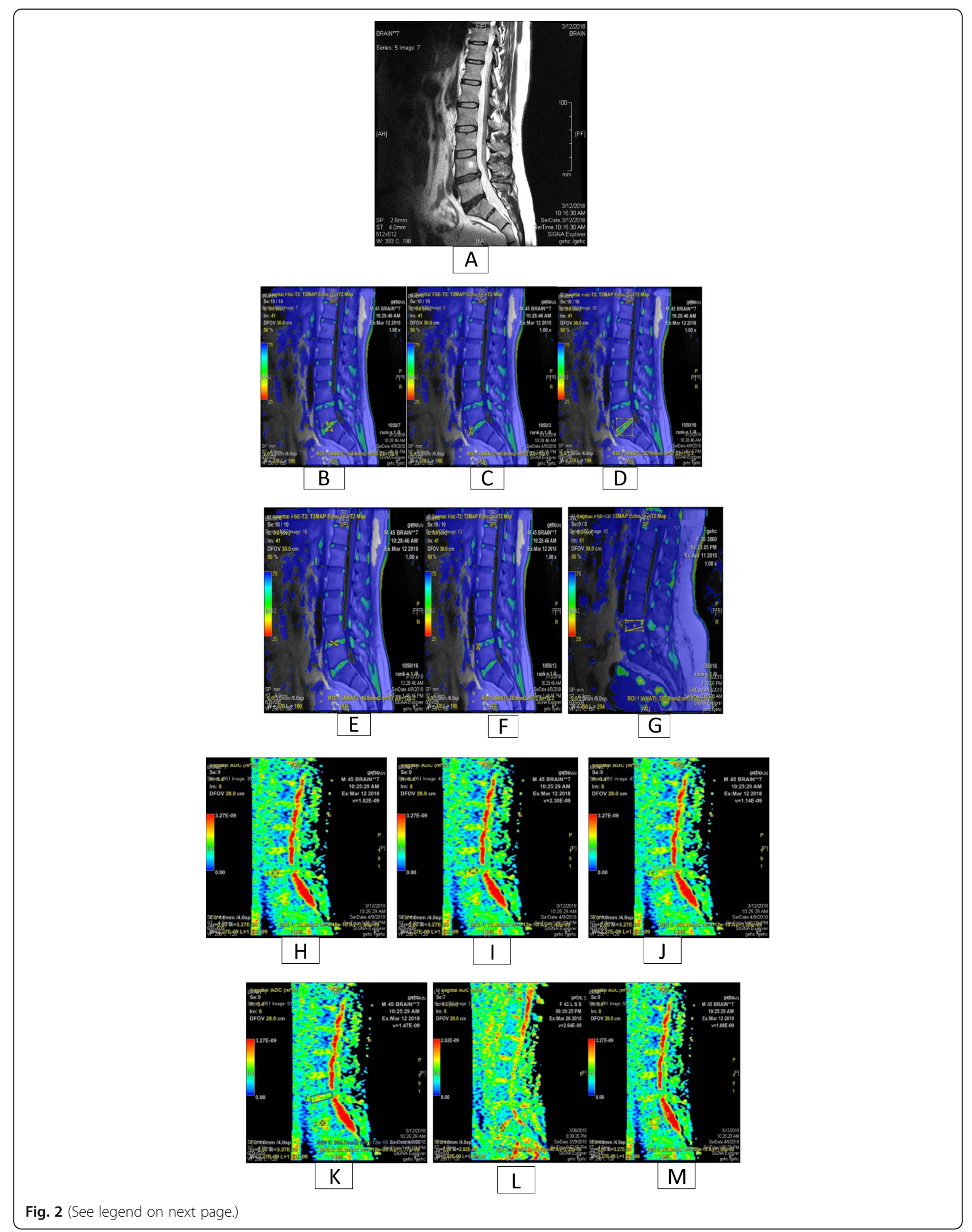


(See figure on previous page.)

Fig. 2 Male patient aged 45 years old presented with low back pain referred to left leg for 6 months. a Sagittal T2Wl of lumbar spine showing: L4/L5 heterogeneous, intermediate to hypointense signal, lost nucleus-annulus distinction, moderately decreased disk space (grade 4). L5/S1 heterogeneous, intermediate to hypointense signal, lost nucleus-annulus distinction, moderately decreased disk space (grade 4). Sagittal color coded post processing T2 mapping (b-d) and (e- $\mathbf{g})$ of most degenerated disks L4-5 and L5-S1 respectively, Sagittal post processing ADC mapping $(\mathbf{h}-\mathbf{j})$ and $(\mathbf{k}-\mathbf{m})$ of same respectively with ROls placed on nucleus pulposus, annulus fibrosus and entire of the disks and the values tabulated as follows:

\begin{tabular}{|c|c|c|c|c|c|c|}
\hline & \multicolumn{3}{|c|}{ T2 mapping } & \multicolumn{3}{|c|}{ ADC values } \\
\hline & NP & $\mathrm{AF}$ & Entire of disk & NP & $\mathrm{AF}$ & Entire of disk \\
\hline L1/L2 & 101 & 81 & 92 & 1.60 & 1.73 & 1.46 \\
\hline L2/L3 & 100 & 75 & 87 & 1.57 & 1.89 & 1.69 \\
\hline L3/L4 & 108 & 85 & 88 & 1.91 & 1.71 & 1.79 \\
\hline L4/L5 & 70 & 66 & 70 & 1.42 & 1.68 & 1.59 \\
\hline$L 5 / S 1$ & 68 & 61 & 64 & 1.12 & 1.75 & 1.66 \\
\hline
\end{tabular}

comparing grades I to $\mathrm{V}$ with $p<0.005$ using one-way ANOVA as seen in Table 1 and Fig. 2.

\section{Correlation between PG and different parameters (T2 mapping and DWI)}

Correlation test showed a moderate negative correlation between Pfirrmann grade and T2 relaxation rates in the nucleus pulposus and entire of the disk; nucleus pulposus NP ( $\mathrm{rs}=-0.583$ ) and entire of the disk ( $\mathrm{rs}=$ - 0.472). Weak negative correlation was also noted between Pfirrmann grade and $\mathrm{T} 2$ relaxation rates in annulus fibrosus $(\mathrm{rs}=-0.214)$.

Regarding ADC mapping, negative correlation between Pfirrmann grade and ADC values in the nucleus pulposus and entire of the disk, NP ( $r s=-0.427)$ and entire of the disk ( $\mathrm{rs}=-0.448)$. Weak negative correlation was also noted between Pfirrmann grade and ADC values in annulus fibrosus ( $\mathrm{rs}=-0.199)$ as shown in Table 2 and Figs. 3 and 4.

Table 2 Correlation between PG and different parameters $(n=$ 300)

\begin{tabular}{lll}
\hline & $\mathrm{PG}$ & \\
\cline { 2 - 3 } & $r_{\mathrm{s}}$ & \\
\hline T2 mapping & -0.583 & $<0.001^{*}$ \\
Central & -0.214 & $0.009^{*}$ \\
Peripheral & -0.472 & $<0.001^{*}$ \\
All & & \\
Diffusion & -0.427 & $<0.001^{*}$ \\
Central & -0.199 & $0.014^{*}$ \\
Peripheral & -0.448 & $<0.001^{*}$ \\
All & & \\
\hline rs: Spearman coefficient & &
\end{tabular}

\section{Correlation of $\mathrm{T}_{2}$ and $\mathrm{ADC}$ values with age}

There is a significant negative correlation for both T2 and ADC values in nucleus pulposus $(p=0.002$ and $p=$ 0.018 ; respectively) and entire of the disk ( $p=0.015$ and $p=0.16$; respectively) with no significant correlation between age and values in annulus fibrosus of both $(p=$ 0.172 and $p=0.494$; respectively) as shown in Table 3 .

\section{The ROC analyses of T2 mapping and DWI}

Receiver-operating characteristic analysis (ROC) between each grade in the nucleus pulposus with the cut-off values of T2 values as shown in Table 4 and Figs. 5, 6, 7, and 8 .

Receiver-operating characteristic analysis between each grade in the nucleus pulposus with the cut-off values of ADC values was shown in Table 5 and Figs. 5, 6, 7, and 8.

\section{Discussion}

Pfirrmann scoring system for classification of degree of disk degeneration based on sagittal T2WI $[5,15]$ was commonly used as a standard in research and clinical applications [16, 17]. It was a subjective estimation and considered as a semi-quantitative assessment of IVDD in vivo. Our study aimed for more quantitative evaluation of IVDD depending upon the biochemical changes in disks by correlation between the Pfirrmann grades and both T2 mapping and ADC values to define which technique is more sensitive in detecting the early stage of IVDD.

Those new techniques were applied to assess patients with lumbar disk degeneration at different stages. Both T2 mapping and ADC maps were acquired for central $(\mathrm{NP})$, peripheral (AF), and entire of the disk then analyzed separately; images were acquired in a sagittal plane so that the five lumbar disks were analyzed in a single plane. 


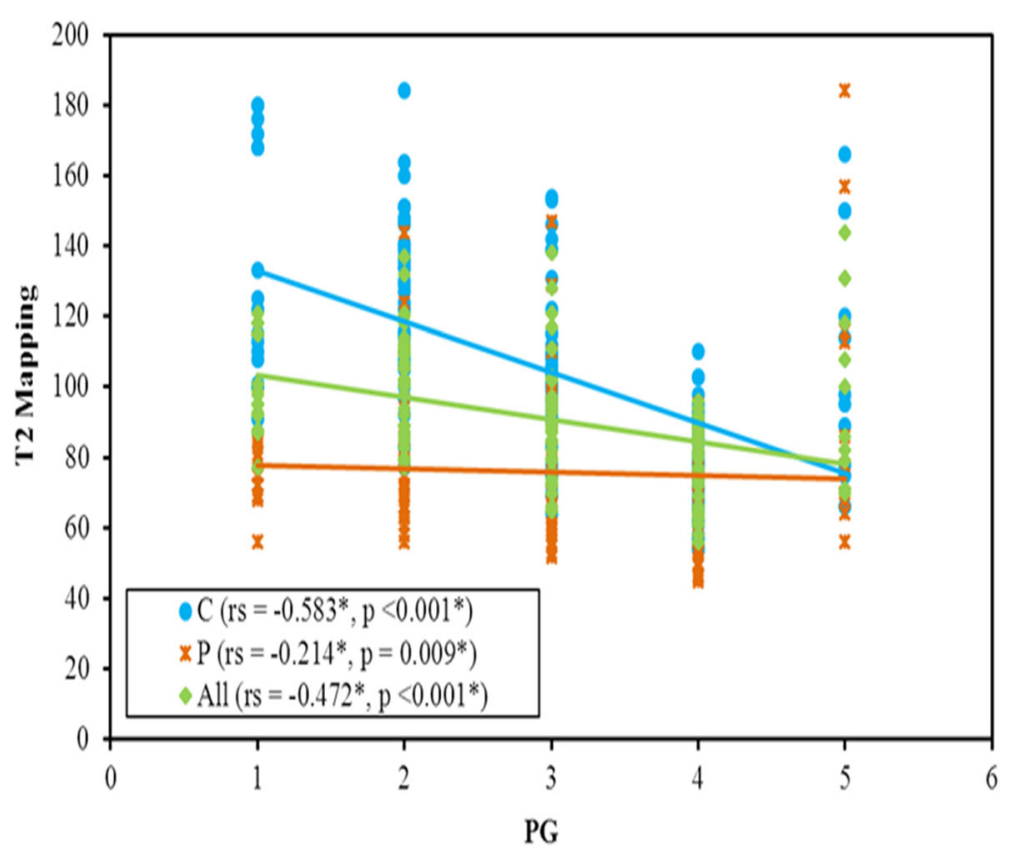

Fig. 3 Correlation between PG and T2 mapping ( $n=300)$

The T2 value in IVD was mainly affected by both rotational and translational motion of water molecules in the collagen matrix $[10,18,19]$. On the other hand, ADC value was sensitive to water content in tissues but did not detect the translational motion of protons at the microscopic level [20]. Thus, ADC value may not be as accurate as $\mathrm{T} 2$ relaxometry in evaluating water and collagen content, although it indirectly allowed evaluation of disk-matrix integrity [21,22].

In our work, we found that T2 mapping and ADC values in the central and all disk values were significantly lower between the IVDD patients and volunteers ( $p$ value $<0.01$ ) while no significant difference in the peripheral portions; this matched with the results of Huang

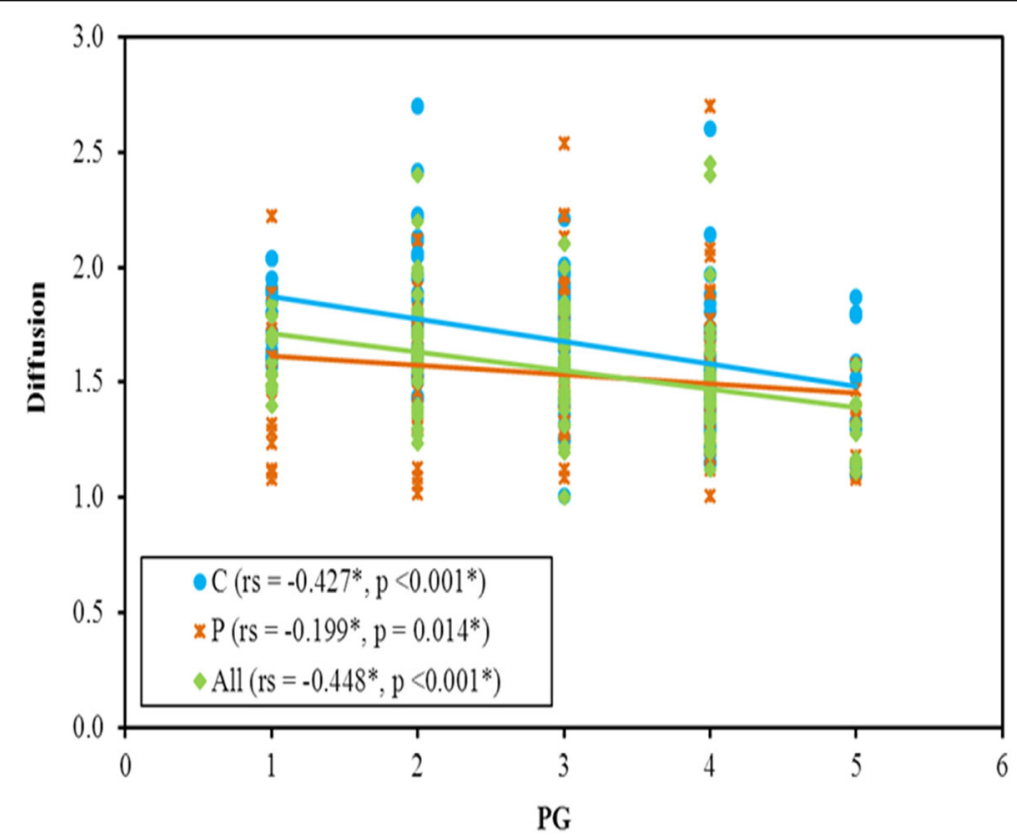

Fig. 4 Correlation between PG and diffusion $(n=300)$ 
Table 3 Correlation between age and different parameters $(n=300)$

\begin{tabular}{lll}
\hline & Age & \\
\cline { 3 - 3 } & $r$ & $p$ \\
\hline T2 mapping & -0.255 & $0.02^{*}$ \\
Central & -0.112 & 0.17 \\
Peripheral & -0.198 & $0.01^{*}$ \\
All & & \\
Diffusion & -0.193 & $0.01^{*}$ \\
Central & -0.056 & 0.49 \\
Peripheral & -0.196 & $0.01^{*}$ \\
All & &
\end{tabular}

rs: Spearman coefficient

*Statistically significant at $p \leq 0.05$

et al. [23] that found T2 mapping values of the NP and posterior AF in their patients group were significantly less than those in the control group $(p<0.1)$ while the value of the anterior AF was similar between the patients and the controls $(p>0.05)$.

The mean age of examined patients in this study was 47.5 years old while in the study reported by Zhao $\mathrm{F}$ et al. [24], the mean age of affection was 54.4 years old. No significant difference in our study between males and females was found; this result was matched with a study done by Xiong $\mathrm{X}$ et al. [25].

In this study, percentage of disks was classified into Pfirrmann grade I $(11.3 \%)$, grade II $(24 \%)$, grade III (28.7\%), grade IV (28.7\%), grade V (7.3\%) while Stelzeneder D et al. [26] reported that six disks (1.8\%) were classified as grade I, $189(57.3 \%)$ as grade II, $96(29.1 \%)$ as grade III, $38(11.5 \%)$ as grade IV, and one disk (0.3\%) as grade $\mathrm{V}$.

The mean T2 values in our study in NP were higher than the means of $\mathrm{T} 2$ values of AF; this is symmetrical with the study done by Shen S et al. [27] who explained that by higher contents of water and proteoglycan [28].

Our study stated that T2 relaxometry values were found inversely proportional to the extent of disk degeneration except in grade $\mathrm{V}$ it was found to be increased again; this is in concordance with the study by Niu G et al. [29]. Pandit P et al. [30] explained that as disk degeneration causes a decrease of glycosaminoglycan which leads to loss of water and hence dehydration of the disk and these effects were more evident at NP compared to AF. The mean T2 value of the NP region in our study ranged from $133.06 \pm 32.85$ to $109.18 \pm$ $33.82 \mathrm{~ms}$, normal. This agreed with Niu G et al. [29]. That reported values ranged from $166 \pm 23$ to $59 \pm 10$ ms normal to degenerated disks respectively. The difference in values may be due to difference of age of population selected in this study, so there was a negative correlation between $\mathrm{T} 2$ values and semi-quantitative grading of disk degeneration which matched with the results of Marinelli NL et al. [20]:

There was also difference in the degree of correlation in NP $(r=-0.583 ; p \leq 0.001)$, AF $(r=-0.214 ; p=$ $0.009)$ and entire of the disk $(r=-0.472 ; p \leq 0.001)$ in concordance with previous studies which showed negative relationship between T2 values and disk degeneration [20, 31, 32]. Marinelli NL et al. explained these variations due to biochemical compositions and histologic changes with degeneration, reflected with differences in the degree of correlation in each region; upon this negative correlation, we conclude that a T2 value of nucleus pulposus is more sensitive than annulus fibrosus and entire of the disk.

The area under the curve values (AUC) for T2 in our study were all within the moderate accuracy range (0.7$0.9)$ except grade I:II that was indicating that T2 valuebased grade scale is useful for evaluation of degenerative degree of intervertebral disk with high degree of objectivity in comparison to traditional Pfirrmann grading system based on visual assessment.

In our literature, we studied T2 cut-off value of NP between grade I and II, which was found to be $\leq 164 \mathrm{~ms}$ with sensitivity $97.2 \%$ and specificity $35.2 \%$. The value between grade II and III was $\leq 104 \mathrm{~ms}$ with sensitivity $60.4 \%$ and specificity $83.3 \%$. Between grade III and IV, the value was $\leq 93 \mathrm{~ms}$ with sensitivity $88.3 \%$ and specificity $65.1 \%$. Between grade IV and V was $>93 \mathrm{~ms}$ with sensitivity $63.6 \%$ and specificity $88.3 \%$ as described before that $\mathrm{T} 2$ values tends to increase in grade $\mathrm{V}$ after decrease in grades from I to IV. Takashima et al. [33] reported that $\mathrm{T} 2$ cut-off value between grade I and II

Table 4 Showing T2 mapping (C) cut off, sensitivity, specificity, AUC, and $p$ value between each grade

\begin{tabular}{lllllllll}
\hline T2 Mapping(central) & AUC & P & $95 \%$ C.I & Cut off & Sensitivity & Specificity & PPV & NPV \\
\hline I:II & 0.549 & 0.092 & $0.368-0.730$ & $\leq 164$ & 97.22 & 35.29 & 76.1 & 85.7 \\
II:II & 0.749 & $<0.001^{*}$ & $0.641-0.857$ & $\leq 104$ & 60.47 & 83.33 & 81.2 & 63.8 \\
III:IV & 0.819 & $<0.001^{*}$ & $0.731-0.907$ & $\leq 93$ & 88.37 & 65.12 & 71.7 & 84.8 \\
IV:V & 0.798 & $0.002^{*}$ & $0.637-0.960$ & $>93$ & 63.64 & 88.37 & 58.3 & 90.5 \\
\hline
\end{tabular}

AUC area under a curve, $p$ value probability value, $C l$ confidence intervals, $N P V$ negative predictive value, $P P V$ positive predictive value *Statistically significant at $p \leq 0.05$ 


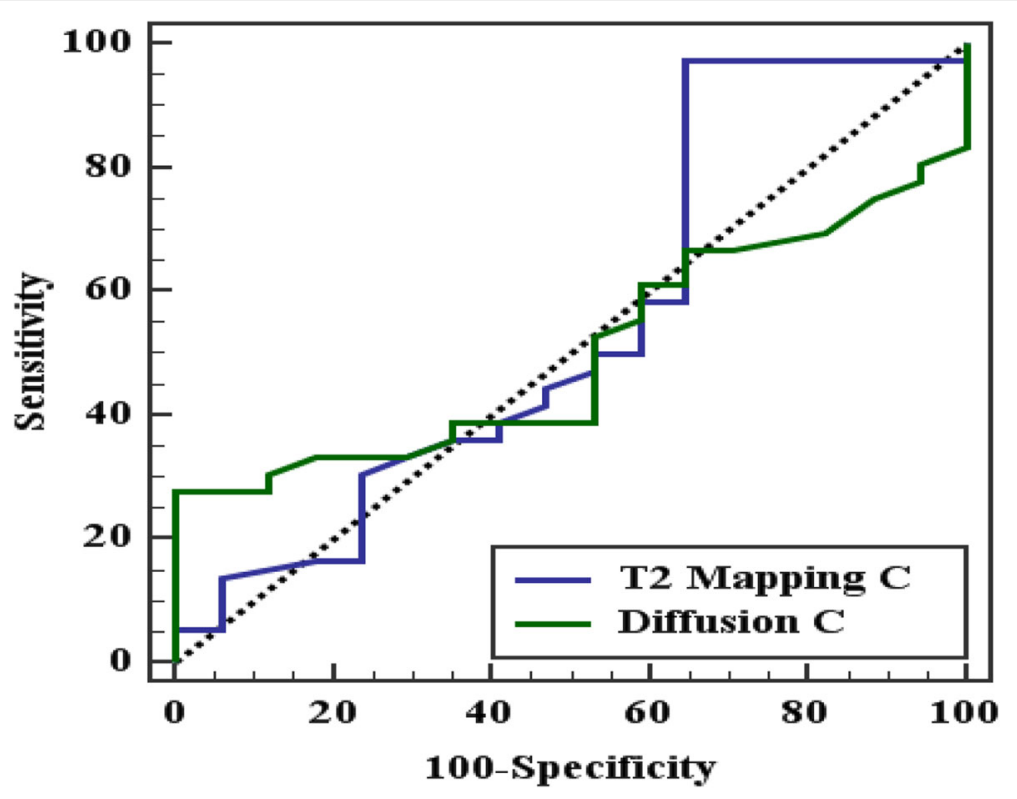

Fig. 5 ROC curve between grade I and grade II

was found to be $116.8 \mathrm{~ms}$, between grades II and III was $>92.7 \mathrm{~ms}$, between grades III and IV was $>72.1 \mathrm{~ms}$, and between grades IV and V was $72 \mathrm{~ms}$.

$\mathrm{ADC}$ is a quantitative parameter calculated from diffusion-weighted imaging, is sensitive in detecting the diffusibility in tissue at molecular level [34]. Previous studies had demonstrated that decreased diffusion was associated with a decrease in nutrient supply in IVDD [12], so ADC mapping could help to reflect the early degenerative changes of disk composition and any subtle changes in its integrity [35]; however, the signal intensity of T2WI in the disks were found to correlate with the ADC values [16].

Our study result for the mean ADC values in NP was $(1.82 \pm 0.29$ and $1.72 \pm 0.25 \mathrm{~ms})$ for grades II and III respectively that were matched with those reported by $\mathrm{Yu}$ H.J et al. [36] who found mean ADC in NP $(1.89 \pm 0.08$ and $1.65 \pm 0.2$ for grade II and III respectively); this difference may arise from that ADC values tend to decrease with the increase of the Pfirrmann grade and age in our study. In our study, mean ADC values in the NPs were lower than those reported in studies by Kealey SM et al.

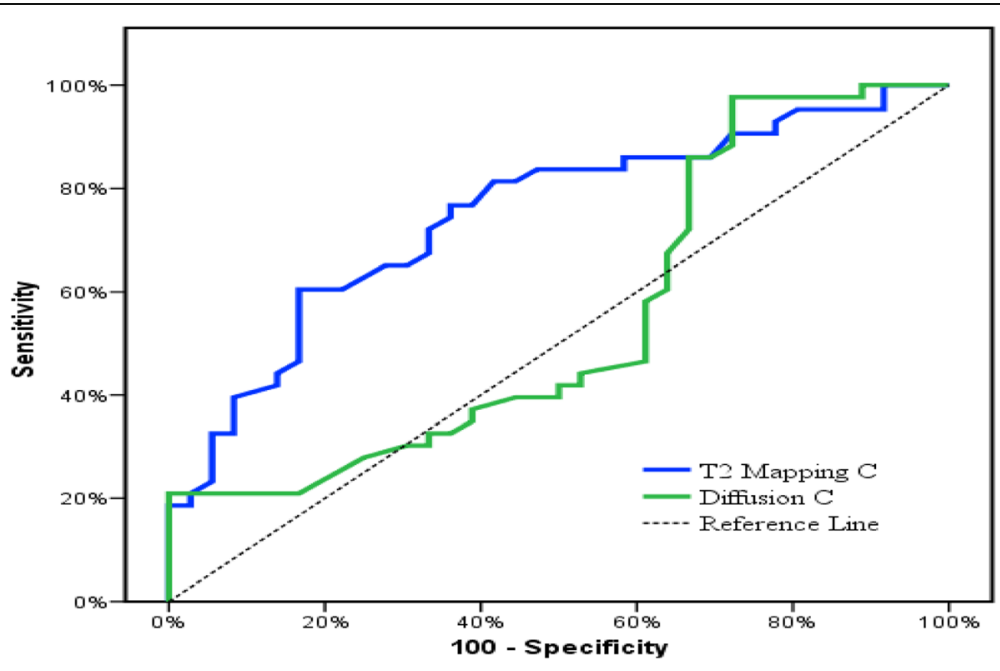

Fig. 6 ROC curve between grade II and grade III 


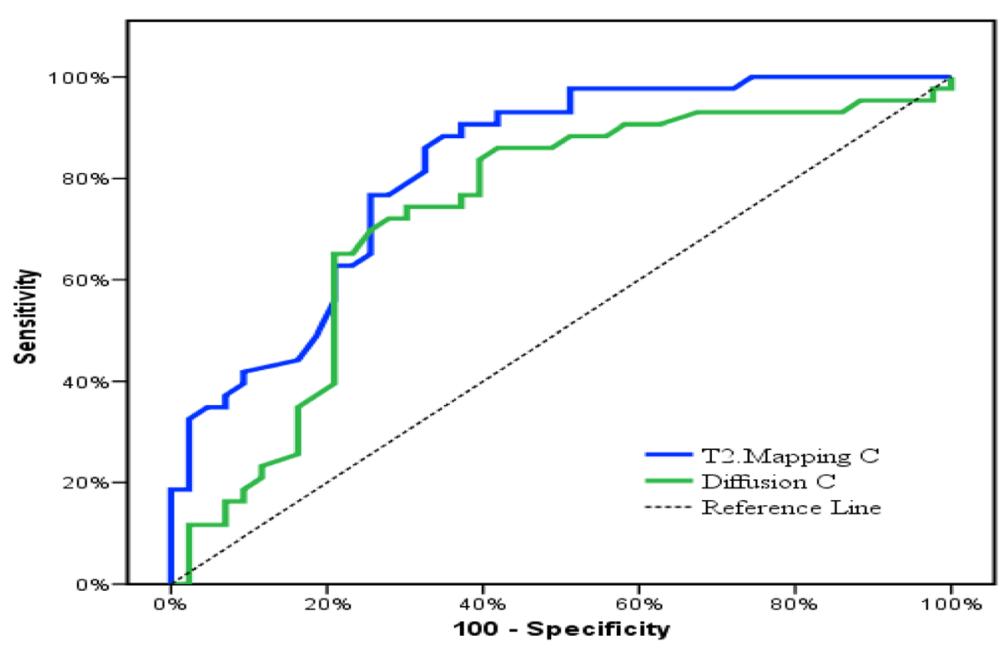

Fig. 7 ROC curve between grade III and grade IV

[37] and Niinimaki J et al. [16] who used also the sagittal plane for diffusion-weighted imaging that may be due to different age of group population.

The ADC values in grade IV and V in this study were more than what $\mathrm{Yu} \mathrm{H}$.J et al. detected [36]; this might be due to dissimilar composition of the patient population.

Niu G et al. [29], Yu H.J et al. [36], and Chen P et al. [38] found a negative correlation between ADC values and Pfirmann grade and were explained by the changes occurring during degeneration which reduce water diffusion in NP and lower ADC values, since the ADC value was highly sensitive to changes of water molecules diffusibility which is in concordance with our study.

There was a difference in the degree of correlation in $\mathrm{NP}, \mathrm{AF}$, and entire of the disk in our study with " $r=$
$-0.427, r=-0.199, r=-0.448$ " respectively that agree with study done by Niu G, et al. [29] who explained these variations due to biochemical compositions and histologic changes with degeneration, reflected with differences in the degree of correlation in each region.

The area under the curve (AUC) for ADC values in our study were all within the low accuracy range (0.50.7 except grade III:IV showing moderate accuracy $(0.7-0.9)$ indicating that ADC value-based grade scale is useful for evaluation of degenerative degree of the intervertebral disk. There was weak negative correlation between age and T2 values of NP and entire of disk with $(r=-0.255, r=-0.198)$ respectively with $p$ value $(0.002,0.015)$ for NP and entire of disk respectively; this matched with Menezes-Reis R et al. [39]. That found

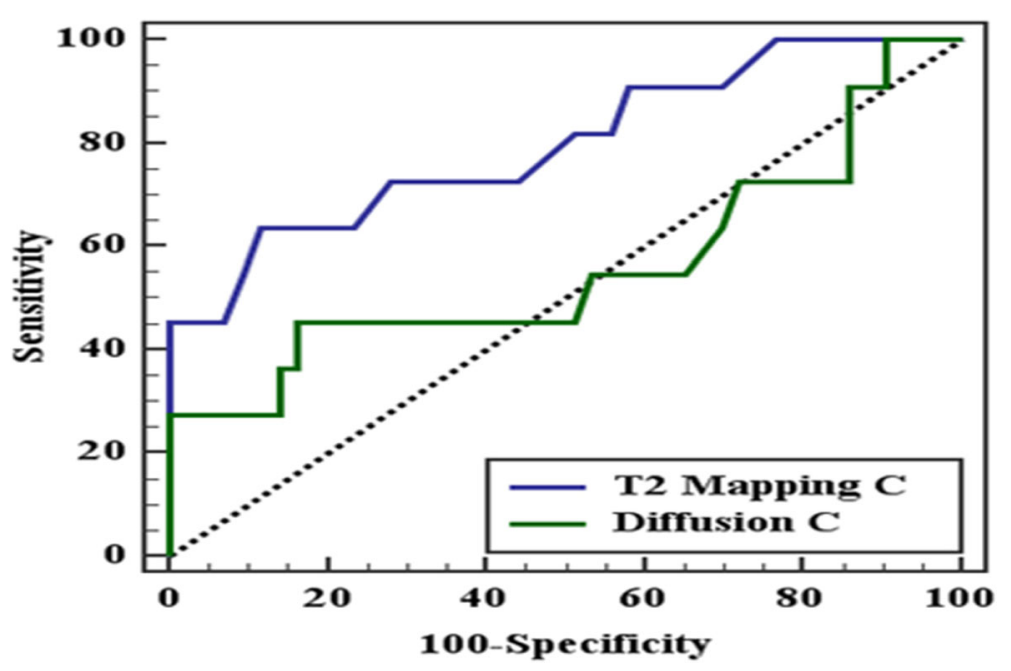

Fig 8 ROC curve between grade IV and grade $V$ 
Table 5 Showing diffusion (C) cut off, sensitivity, specificity, AUC, and $p$ value between each grade

\begin{tabular}{lllllllll}
\hline Diffusion (Central) & AUC & P & $95 \%$ C.I & Cut off & Sensitivity & Specificity & PPV & NPV \\
\hline I:II & 0.502 & 0.568 & $0.270-0.632$ & $>1.97$ & 27.78 & 88.24 & 83.3 & 36.6 \\
II:II & 0.549 & 0.457 & $0.417-0.681$ & $\leq 2.01$ & 97.67 & 27.78 & 56.8 & 80.0 \\
III:IV & 0.730 & $<0.001^{*}$ & $0.618-0.841$ & $\leq 1.55$ & 65.12 & 79.07 & 75.7 & 69.4 \\
IV:V & 0.560 & 0.540 & $0.335-0.785$ & $\leq 1.33$ & 45.45 & 83.72 & 41.7 & 85.7 \\
\hline
\end{tabular}

AUC area under a curve, $p$ value probability value, $C l$ confidence interval, $N P V$ negative predictive value, $P P V$ positive predictive value

*Statistically significant at $p \leq 0.05$

correlation between age and T2 relaxation time $(r=$ $-0.30, p<0.0001)$ with average age of participants $27.1 \pm 4.8$ years old.

Marinelli NL et al. [18], Niu G et al. [29], Wang W et al. [40], Boos $\mathrm{N}$ et al. [41], and Leung VY et al. [42] analyzed the correlation between the T2 and ADC values and age and reached the result that all the T2 values and $\mathrm{ADC}$ values were significantly decreased with the increase of age and spearman correlation coefficient of -0.594 and -0387 were demonstrated respectively. This were matched with our results with spearman correlation coefficient of -0.472 and -0.448 were demonstrated respectively. These results indicate when compared to ADC that $\mathrm{T} 2$ is a more sensitive and advanced measure to quantitatively delineate the early stage and age-related changes in IVDD.

The main result in our current study was that T2 and ADC values differ chiefly in their respective ability to discriminate Pfirrmann grades, as manifested by a completely different area under the ROC curves. Moreover, the T2 measure is more sensitive in differentiating the early stages of disk degeneration especially between grades I and III.

The major limitations in our study were absence of histological correlation with the results, absence of direct relation between the morphological changes of disks and T2 mapping and DWI, also subjective grading of disks by Pfirrmann grading

\section{Conclusion}

Our results claimed that T2 relaxometry and ADC values in degenerated lumbar disks negatively correlated with the Pfirrmann grades. More importantly, T2 provides the most sensitive and advanced MR imaging measure for detecting the early stage and aging-related changes in the disks quantitatively; $\mathrm{T}_{2}$ relaxometry and $\mathrm{ADC}$ maps are highly recommended to be added to conventional MRI sequences for all patients complaining of low-back pain for early detection of disk degeneration and follow-up.

\section{Abbreviations}

ADC: Apparent diffusion coefficient; AF: Annulus fibrosus; AUC: Area under the curve; DDD: Degenerative disk disease; DWl: Diffusion-weighted image; FSE: Fast spin-echo; IVD: Intervertebral disk; IVDD: Intervertebral disk degeneration; MR: Magnetic resonance; NP: Nucleus pulposus; PG: Pfirrmann grades; ROI: Region of interest; SE: Spin-echo; SE-EPI: Spin-echo echo-planar imaging

\section{Acknowledgements}

To all the participants for their cooperation and patience.

\section{Competing of interests}

The authors declare that they have no competing of interests.

\section{Authors' contributions}

RA suggested the research idea, ensured the original figures and data in the work, minimized the obstacles to the team of work, correlated the study concept and design, and had the major role in analysis. MD supervised the study with significant contribution to design the methodology, manuscript revision, and preparation. AAb correlated the clinical data of patient and matched it with the findings, drafted, and revised the work. AM collected data in all stages of manuscript, performed data analysis. All authors read and approved the final manuscript.

\section{Funding}

No funding. Not applicable for this section.

\section{Availability of data and materials}

The authors' confirm that all data supporting the finding of the study are available within the article and the raw data supporting the findings were generated and available at the corresponding author on request.

\section{Ethics approval and consent to participate}

Informed written consents were taken from the patients and healthy volunteers. The study was approved by ethical committee of Tanta university hospital, faculty of medicine. Number 4563-2019.

\section{Consent for publication}

All participants included in the research gave written consent to publish the data included in the study. Authors accepted to publish the paper.

\section{Author details}

${ }^{1}$ Radiology and Medical Imaging Department, Faculty of Medicine, Tanta University, Tanta, Egypt. ${ }^{2}$ Neuropsychiatric Departement, Faculty of Medicine, Tanta University, Tanta, Egypt.

Received: 8 July 2019 Accepted: 6 September 2019

Published online: 21 October 2019

\section{References}

1. Andersson GB (1998) Epidemiology of low back pain. Acta Orthop Sc and Suppl 281:28-31

2. Roh JS, Teng AL, Yoo JU et al (2005) Degenerative disorders of the lumbar and cervical spine. Orthop Clin North Am 36(3):255-262

3. Schellhas K (1996) Lumbar disc high-intensity zone- MRI \& Discography. Spine 21:79-86

4. Videman T, Battie MC, Gibbons LE et al (2017) A new quantitative measure of disc degeneration. Spine J 17:746-753

5. Pfirmann CWA, Metzdrof A, Zanetti M et al (2001) Magnetic resonance classification of lumbar intervertebral disc degeneration. spine 26:1873-1878 
6. Wang C, Mcardle E, Fenty M et al (2010) Validation of sodium magnetic resonance imaging of intervertebral disc. Spine 35:505-510

7. Habibi Z, Maleki F, Meybodi AT et al (2014) Lumbosacral sagittal alignment in association to intervertebral disc diseases. Asian Spine J 8:813-819

8. Modic MT, Ross JS (2007) Lumbar degenerative disk disease. Radiology 245:43-61

9. Wang YXJ, Griffith JF, Leung JCS et al (2014) Age related reduction of T1rho and T2 magnetic resonance relaxation times of lumbar intervertebral disc. Quant Imaging Med Surg 4:259-264

10. Perry J, Haughton V, Anderson PA et al (2006) The value of T2 relaxation times to characterize lumbar intervertebral disks: preliminary results. AJNR 27:337-342

11. Kim DJ, Suh JS, Jeong EK et al (1999) Correlation of laminated MR appearance of articular cartilage with histology, ascertained by artificial landmarks on the cartilage. J Magn Reson Imaging 10:57-64

12. Nguyen-minh C, Ho KC, Xu R, at al (1997) Effect of degeneration of the intervertebral disc on the process of diffusion. AJNR Am J Neuroradiol 18:435-442.

13. Nguyen-minh C, Haughton VM, Papke RA et al (1998) Measuring diffusion of solutes into intervertebral discs with MR imaging and paramagnetic contrast medium. AJNR Am J Neuroradiol 19:1781-1784

14. Waris $\mathrm{E}$, Eskelin $\mathrm{M}$, Hermunen $\mathrm{H}$ et al (2007) Disc degeneration in low back pain: a 17-year follow-up study using magnetic resonance imaging. Spine (Phila Pa 1976) 32:681-684

15. Griffith JF, Wang YXJ, Antonio GE et al (2017) Modified Pfirmann Grading System for Lumbar Intervertebral Disc Degeneration. Spine 32:708-712

16. Niinimaki J, Korkiakoski A, Ojala O et al (2009) Association between visual degeneration of intervertebral discs and the apparent diffusion coefficient. Magn Reson Imaging 27:641-647

17. Nguyen AM, Johannessen W, Yoder JH et al (2008) Noninvasive quantification of human nucleus pulposus pressure with use of T1 rhoweighted magnetic resonance imaging. J Bone Joint Surg Am 90:796-802

18. Marinelli NL, Haughton VM, Munoz A et al (2009) T2 relaxation times of intervertebral disc tissue correlated with water content and proteoglycan content. Spine (Phila Pa 1976) 34:520-524

19. Trattnig S, Stelzeneder D, Goed S et al (2010) Lumbar intervertebral disc abnormalities: comparison of quantitative T2 mapping with conventional MR at 3.0 T. Eur Radiol 20:2715-2722

20. Marinelli NL, Haughton VM, Anderson PA (2010) T2 relaxation times correlated with stage of lumbar intervertebral disk degeneration and patient age. AJNR Am J Neuroradiol 31:1278-1282

21. Blumenkrantz G, Zuo J, Li X et al (2010) In vivo 3.0-Tesla magnetic resonance T1rho and T2 relaxation mapping in subjects with intervertebra disc degeneration and clinical symptoms. Magn Reson Med 63:1193-1200

22. Kerttula L, Kurunlahti $M$, Jauhiainen J et al (2001) Apparent diffusion coefficients and $\mathrm{T} 2$ relaxation time measurements to evaluate disc degeneration. A quantitative MR study of young patients with previous vertebral fracture. Acta Radiol 42:585-591

23. Huang L, Liu Yuan, , Ding Yi, et al (2017) Quantitative evaluation of lumbar intervertebral disc degeneration by axial T2* mapping .Medicine 96(51):e3939.

24. Wang YXJ, Zhao F, Griffith JF et al (2013) T1 rho and T2 relaxation times for lumbar disc degeneration: an in-vivo comparative study at 3.0 Tesla MRI. European Radiology 23:228-234

25. Xiong X, Zhou Z, Figini M et al (2018) Multi-parameter evaluation of lumbar intervertebral disc degeneration using quantitative magnetic resonance imaging techniques. American Journal of Translational Research 10:444-454

26. Stelzeneder D, Welsch GH, Kovacs BK et al (2012) Quantitative T2 evaluation at $3.0 \mathrm{~T}$ compared to morphological grading of the lumbar intervertebral disc: a standardized evaluation approach in patients with low back-pain. Eur J Radiol 81:324-330

27. Shen $\mathrm{S}$, Wang $\mathrm{H}$, Zhang J et al (2016) Diffusion weighted imaging, diffusion tensor imaging and $\mathrm{T}^{*}$ mapping of lumbar intervertebral disc in young healthy adults. Iran J Radiol 13(1):e30069

28. Urban JP, Roberts S (2003) Degeneration of the intervertebral disc. Arthritis Res 5:120-130

29. Niu G, Yang J, Wang R et al (2011) MR Imaging Assessment of Lumbar Intervertebral Disk Degeneration And Age -Related Changes: Apparent Diffusion Coefficient versus T2 Quantitation. Am J Neuroradiol 32:1617-1622

30. Pandit P, Talbott FJ et al (2016) T1 and T2 based characterization of regional variations in intervertebral discs to detect early degenerative changes. J Orthopedic Res 34:1373-1381
31. Watanabe A, Benneker LM, Boesch C et al (2007) Classification of intervertebral disk degeneration with axial T2 mapping. Am J 189:936-942

32. Yoon MA, Hong SJ, Kang CH et al (2016) T1 rho and T2 mapping of lumbar intervertebral disc: correlation with degeneration and morphologic changes in different disc regions. Magnetic resonance imaging 34:932-939

33. Takashima H, Takebayashi T, Yoshimoto M et al (2012) Correlation between T2 relaxation time and intervertebral disc degeneration. Skeletal Radiol 41:163-167

34. Grunhagen T, Shirazi-Adl A, Fairbank J.C, et al (2011) Intervertebral disc nutrition: a review of factors influencing concentrations of nutrients and metabolites. Orthop Clin North Am 42: 465-477.

35. Antoniou J, Demers CN, Beaudoin G et al (2004) Apparent diffusion coefficient of intervertebral discs related to matrix composition and integrity. Magn Reson Imaging 22:963-972

36. Yu HJ, Bahri S, Gardner V et al (2015) In vivo quantification of lumbar disc degeneration: assessment of $A D C$ value using a degenerative Scoring system based on Pfirmann framework. Eur Spine J 24:2442-2448

37. Kealey SM, Aho T, Delong D et al (2005) Assessment of apparent diffusion coefficient in normal and degenerated intervertebral lumbar disks: initial experience. Radiology 235:569-574

38. Chen P, Wu C, Huang M et al (2018) Apparent diffusion coefficient of diffusion-weighted imaging in evaluation of cervical intervertebral disc degeneration: an observational study with $3 \mathrm{~T}$ magnetic resonance Imaging. Biomed Research International 1-7. https://doi.org/10.1155/2018/6843053

39. Menezes-Reis R, Salmon CE, Carvalho CS et al (2015) T1 rho and T2 mapping of the intervertebral disc: comparison of different methods of segmentation. AJNR Am J Neuroradiology 36:606-611

40. Wang W, Hou J, Lv D et al (2017) Multimodal quantitative magnetic resonance imaging for lumbar intervertebral disc degeneration. Exp Ther Med 14:2078-2084

41. Boos N, Weissbach S, Rohrbach $\mathrm{H}$ et al (2002) Classification of age-related changes in lumbar intervertebral discs: 2002 Volvo Award in basic science. Spine (Phila Pa 1976) 27:2631-2644

42. Leung VY, Hung SC, Li LC et al (2008) Age-related degeneration of lumbar intervertebral discs in rabbits revealed by deuterium oxide-assisted MRI. Osteoarthritis Cartilage 16:1312-1318

\section{Publisher's Note}

Springer Nature remains neutral with regard to jurisdictional claims in published maps and institutional affiliations.

\section{Submit your manuscript to a SpringerOpen ${ }^{\circ}$ journal and benefit from:}

- Convenient online submission

- Rigorous peer review

- Open access: articles freely available online

High visibility within the field

- Retaining the copyright to your article

Submit your next manuscript at $\boldsymbol{\nabla}$ springeropen.com 